\title{
Equation of State at Finite Density from Imaginary Chemical Potential
}

\author{
Tetsuya Takaishi* \\ Hiroshima University of Economics \\ Hiroshima 731-0192 JAPAN \\ E-mail: takaishi@hiroshima-u.ac.jp
}

\section{Philippe de Forcrand}

Institut für Theoretische Physik, ETH Zürich, CH-8093 Zürich, SWITZERLAND

and

CERN, Physics Department, TH Unit, CH-1211 Geneva 23, SWITZERLAND

E-mail: forcrandephys.ethz.ch

\section{Atsushi Nakamura}

Hiroshima University

Higashi-Hiroshima 739-8521 JAPAN

E-mail: nakamura@riise.hiroshima-u.ac.jp

We perform two flavor QCD simulations with an imaginary chemical potential and measure derivatives of the pressure up to 4th order as a function of the imaginary chemical potential and the temperature $T \in\left[0.83 T_{c}, 2 T_{c}\right]$. For temperatures $T \geq T_{c}$, these derivatives are fitted by a Taylor series in $\mu / T$ about $\mu=0$. A fit limited to 4 th order describes the data poorly at all temperatures, showing that we are sensitive to 6th order contributions. Similarly, a 6th order fit fails for temperatures $T_{c} \leq T \leq 1.05 T_{c}$, showing the need for 8th order terms. Thus, our method may offer a computational advantage over the direct measurement of Taylor coefficients at $\mu=0$. At temperatures $T \leq T_{c}$, we fit our data with a hadron resonance gas ansatz. The fit starts to fail at $T \gtrsim 0.95 T_{c}$. Using our fits, we also reconstruct the equation of state as a function of real quark and isospin chemical potentials.

The XXVII International Symposium on Lattice Field Theory - LAT2009

July 26-31 2009

Peking University, Beijing, China

\footnotetext{
*Speaker.
} 


\section{Introduction}

Although lattice QCD has been used successfully for simulations at zero and finite temperatures and at zero density, Monte Carlo simulations at non-zero densities suffer from a technical problem: the lattice QCD action becomes complex, which prevents its customary probabilistic interpretation. In principle one could perform simulations at zero density, and use the reweighting technique to obtain information at finite densities. An early attempt known as the Glasgow method [1] did not work due to the overlap problem: the configurations at zero density were too "far" from the target configurations at non-zero densities. Considerable progress has been accomplished by generalizing the Glasgow method to two-parameter reweighting [2]. Nevertheless, the range of reliability of this technique is difficult to assess, and its failure can go undetected.

Therefore, another, more conservative way to deal with finite baryon densities may be useful. It consists of calculating Taylor coefficients of observables with respect to the chemical potential $\mu$ about $\mu=0$. Those Taylor coefficients can be expressed as expectation values of complicated observables, which can be measured at zero density. Thus, there is no difficulty to perform Monte Carlo simulations in this method. A first, pioneering attempt to obtain quark susceptibilities [3] has been followed by numerous works, obtaining in particular the response of screening masses to chemical potential $[4,5,6]$. The Taylor expansion method has also been used for studies of the equation of state, of the phase transition and of higher order susceptibilities $[7,8,9,10]$. However, the complexity of the observable representing the Taylor coefficient, and the computer effort to measure it, increase rapidly with the order of the Taylor expansion. This motivates us to follow a different strategy.

Since no difficulty appears for simulations at imaginary chemical potential $\mu=i \mu_{I}$, one can obtain information at finite baryon densities by analytic continuation of observables measured at finite $\mu_{I}$. Actually, this imaginary chemical potential strategy has been applied with success to the determination of the phase transition [11].

In this study, we perform simulations at finite $\mu_{I}$ and measure derivatives of the pressure as a function of $\mu_{I}$. These derivatives contain information about the Taylor coefficients of the $\mu=0$ expansion, which can be extracted by fitting. Finally, we try to reconstruct the equation of state at finite baryon and isospin densities. The strategy of our method and preliminary results were presented rather long ago in [12]. Here we report further progress on this project. A related approach, where the quark density is measured at imaginary quark and real isospin chemical potentials and then fitted by a polynomial ansatz, has recently been presented in [15].

\section{Equation of State at Finite Chemical Potential}

The lattice QCD partition function with $N_{f}$ flavors of staggered fermions can be written as

$$
Z=\int \Pi_{i}^{N_{f}} \operatorname{det} M\left(U, m_{i}, \mu_{i}\right)^{1 / 4} \exp \left(-S_{g}[U]\right) d U,
$$

where $S_{g}[U]$ is the gauge action and $M\left(U, m_{i}, \mu_{i}\right)$ stands for the staggered Dirac operator with quark mass $m_{i}$ and chemical potential $\mu_{i}{ }^{1}$. In this study we consider $N_{f}=2$ degenerate fermion species and use the standard Wilson gauge action.

\footnotetext{
${ }^{1}$ We set aside potential problems with "rooting" the determinant, particularly at non-zero chemical potential.
} 
The pressure or the equation of state with chemical potential $\mu_{u}$ and $\mu_{d}$ is given by

$$
p\left(\mu_{u}, \mu_{d}\right)=-\frac{F}{V}=\frac{T}{V} \ln Z\left(\mu_{u}, \mu_{d}\right),
$$

and can be expanded in a Taylor series about $\mu_{u}=\mu_{d}=0$ as

$$
\frac{\Delta p}{T^{4}} \equiv \frac{p\left(\mu_{u}, \mu_{d}\right)-p(0,0)}{T^{4}}=\sum_{n, m=1} \frac{1}{n ! m !} f_{n m}\left(\frac{\mu_{u}}{T}\right)^{n}\left(\frac{\mu_{d}}{T}\right)^{m}
$$

where $f_{n m}$ are the Taylor expansion coefficients. They vanish when $(n+m)$ is odd due to CP symmetry. Furthermore, for equal quark masses there is another symmetry $f_{n m}=f_{m n}$. The $f_{n m}$ 's are related to derivatives $\chi_{i j}$ of the pressure measured at non-zero chemical potential by

$$
T^{i+j-4} \chi_{i j}=\frac{\partial^{i+j}\left(p\left(\mu_{u}, \mu_{d}\right) / T^{4}\right)}{\partial\left(\mu_{u} / T\right)^{i} \partial\left(\mu_{d} / T\right)^{j}}=\sum_{n=i, m=j} \frac{1}{(n-i) !(m-j) !} f_{n m}\left(\frac{\mu_{u}}{T}\right)^{n-i}\left(\frac{\mu_{d}}{T}\right)^{m-j}
$$

While at zero density $\chi_{i j}=f_{i j} T^{4-i-j}$, at non-zero densities $\chi_{i j}$ includes higher order $f_{n m}$ terms, and does not vanish for odd $(i+j)$. This suggests to use all available $\chi_{i j}$ 's at non-zero densities, in order to estimate the $f_{n m}$ 's. Here, we try to estimate $f_{n m}$ by fitting all $\chi_{i j}$ simultaneously to the polynomial expansions eq.(2.4). Of course, $\chi_{i j}$ at non-zero baryon density is not directly obtainable from simulations on the lattice because of the sign problem. However, $\chi_{i j}$ can be obtained through simulations at imaginary quark chemical potential or at real isospin density. Here, we calculate $\chi_{i j}$ at imaginary chemical potentials.

Therefore, we set $\mu=i \mu_{I}$. Each $\chi_{i j}$ depends on higher order Taylor coefficients following eq.(2.4). Therefore, with sufficiently accurate data on $\chi_{i j}$ one can also obtain higher order Taylor coefficients $f_{n m}, n>i, m>j$. The measurements of the derivatives involve computing traces of inverse Dirac matrix products. These traces were estimated using the noise method with $40 Z_{2}$ random vectors. In this study we measure $\chi_{i j}$ up to $i+j=4$. Thus we have 8 different $\chi_{i j}$ 's. We fit all the data to the corresponding 8 polynomial expansions eq.(2.4) truncated to a given order $(n+m)$, and try to obtain the Taylor coefficients $f_{n m}$.

As we will see, in the confined phase a Taylor expansion is not the most compact description of the pressure. Instead, for $T \leq T_{c}$ we use the Hadron Resonance Gas (HRG) model. In the HRG model the pressure is given as ${ }^{2}$

$$
\begin{aligned}
\frac{\Delta p\left(\mu_{u}, \mu_{d}\right)}{T^{4}} & =G\left[\cosh \left(\frac{2 \mu_{I s}}{T}\right)-1\right]+R\left[\cosh \left(\frac{3 \mu_{q}}{T}\right) \cosh \left(\frac{\mu_{I s}}{T}\right)-1\right] \\
& +W\left[\cosh \left(\frac{3 \mu_{q}}{T}\right)\left(\cosh \left(\frac{\mu_{I s}}{T}\right)+\cosh \left(\frac{3 \mu_{I s}}{T}\right)\right)-2\right]
\end{aligned}
$$

where $G, R$ and $W$ are constants related to the hadron spectrum, and quark and isospin chemical potentials $\mu_{q}$ and $\mu_{I s}$ are defined as $\mu_{q}=\left(\mu_{u}+\mu_{d}\right) / 2$ and $\mu_{I s}=\left(\mu_{u}-\mu_{d}\right) / 2$ respectively.

The derivatives of the pressure with respect to $\mu_{u}$ and $\mu_{d}$, instead of having the polynomial form eq.(2.4), are now obtained by differentiating eq.(2.5). The coefficients $G, R$ and $W$ are then

\footnotetext{
${ }^{2}$ This expression is taken from (4.3) in [8].
} 


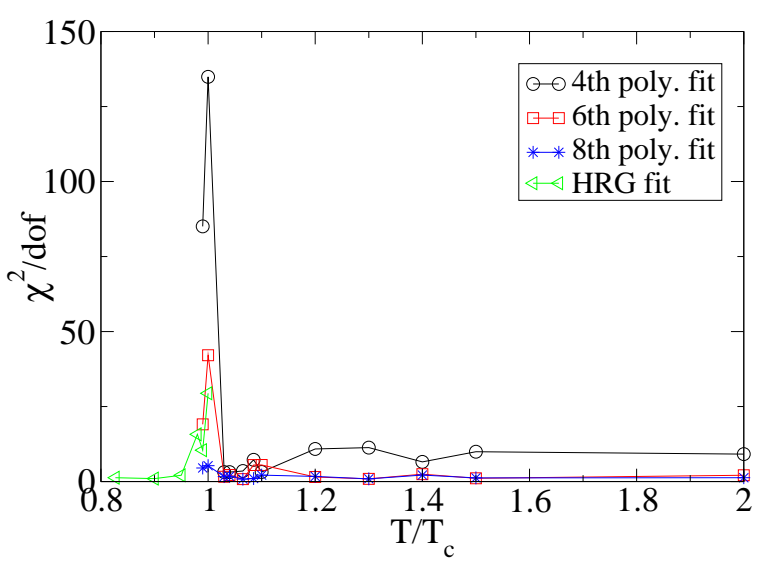

Figure 1: $\chi^{2} / d o f$ of various polynomial ansätze as a function of $T / T_{c}$. The fitting range of $a \mu_{I}$ is 0.0-0.24.

extracted by fitting imaginary- $\mu$ data. In terms of $G, R$ and $W$, the first Taylor coefficients are given by

$$
\begin{gathered}
f_{20}=\left(G+\frac{5}{2} R+7 W\right), \\
f_{11}=-(G+2(R+W)), \\
f_{22}=G+4(R+W), \\
f_{31}=G+\frac{7}{2} R+49 W, \\
f_{40}=-G+5(R+W) .
\end{gathered}
$$

Table 1: $\chi^{2} /$ dof for polynomial ansatz of degree 4,6 and 8 (maximum value of $(n+m)$ in eq.(2.4)).

\begin{tabular}{cclllllllllll}
\hline$T / T_{c}$ & 0.99 & 1.00 & 1.03 & 1.04 & 1.065 & 1.085 & 1.1 & 1.2 & 1.3 & 1.4 & 1.5 & 2.0 \\
\hline 4th & 85.1 & 134.9 & 3.15 & 3.14 & 3.50 & 7.24 & 3.20 & 10.9 & 11.3 & 6.67 & 9.95 & 9.15 \\
6th & 19.1 & 42.1 & 1.60 & 2.19 & 0.82 & 5.50 & 5.53 & 1.52 & 0.89 & 2.46 & 1.09 & 2.10 \\
8th & 4.53 & 5.29 & 1.64 & 1.77 & 0.81 & 1.01 & 2.15 & 1.72 & 0.91 & 2.16 & 1.22 & 1.28 \\
\hline
\end{tabular}

Table 2: $\chi^{2} / d o f$ for HRG ansatz.

\begin{tabular}{ccccccc}
\hline$T / T_{c}$ & 0.83 & 0.9 & 0.95 & 0.98 & 0.99 & 1.00 \\
\hline HRG & 1.29 & 1.00 & 2.10 & 15.8 & 10.5 & 29.4 \\
\hline
\end{tabular}

\section{Simulations at Imaginary Chemical Potential}

We have performed simulations on $8^{3} \times 4$ lattices at a quark mass $m_{q}=0.05$ and imaginary chemical potentials $a \mu_{I}=0.0, \ldots, 0.24$. We have chosen 16 values of $\beta$ ranging from 4.90 to 6.85 , which correspond to $T / T_{c}=0.83 \sim 2.0$. Most of the simulations were performed using the Ralgorithm with a step size $\Delta t=0.02$. We also used the Rational HMC algorithm [13] to check 


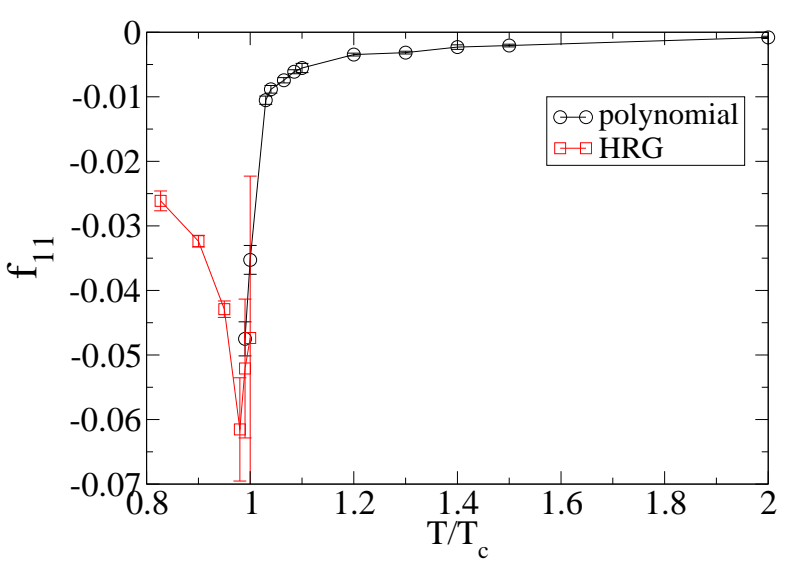

(a)

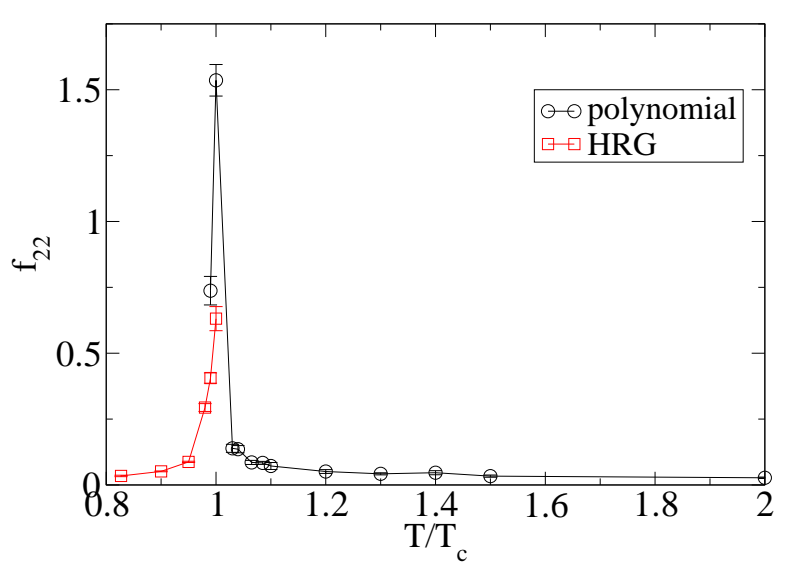

(c)

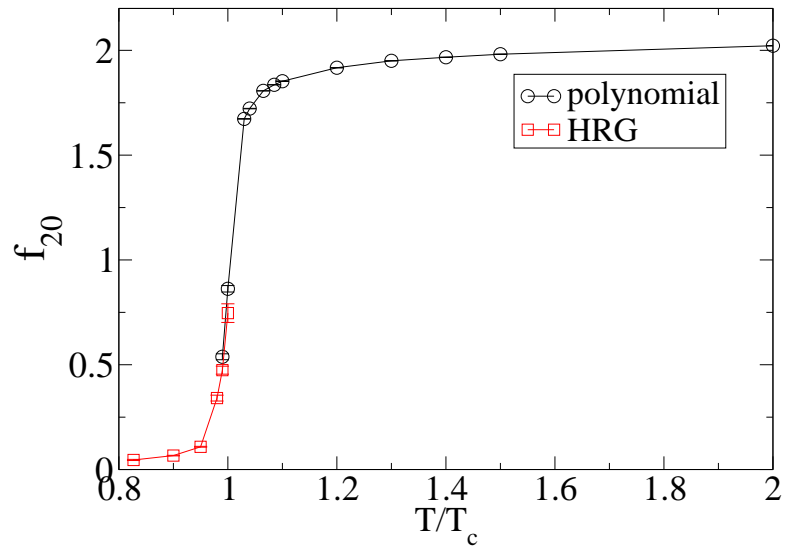

(b)

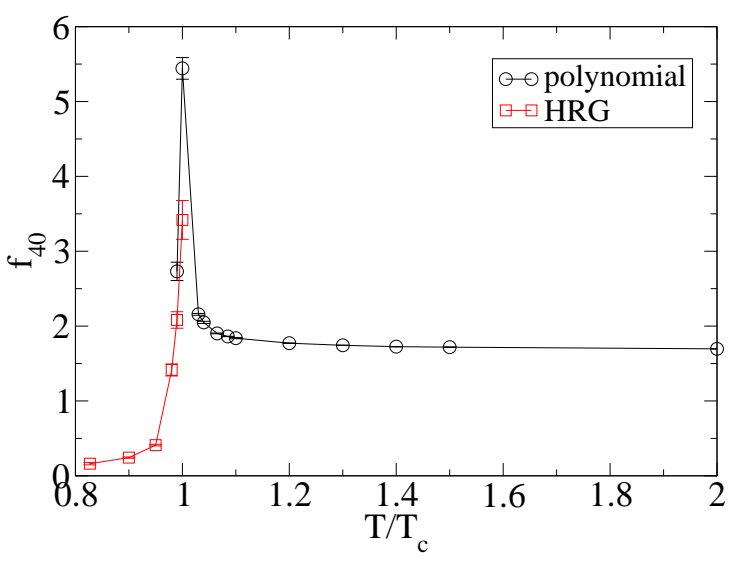

(d)

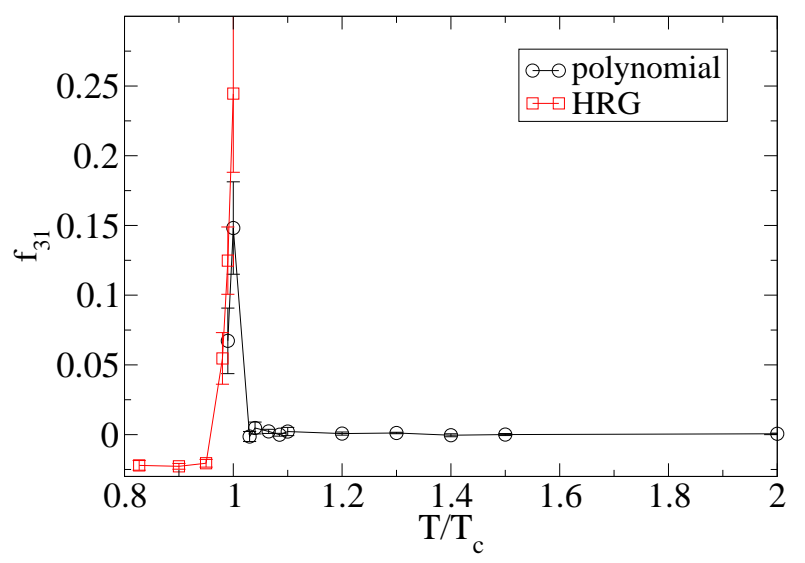

(e)

Figure 2: Taylor coefficients: (a) $f_{11}$, (b) $f_{20}$, (c) $f_{22}$, (d) $f_{40}$ and (e) $f_{31}$. 


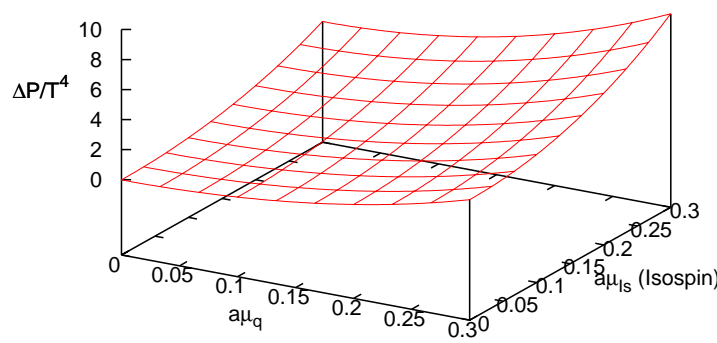

(a)

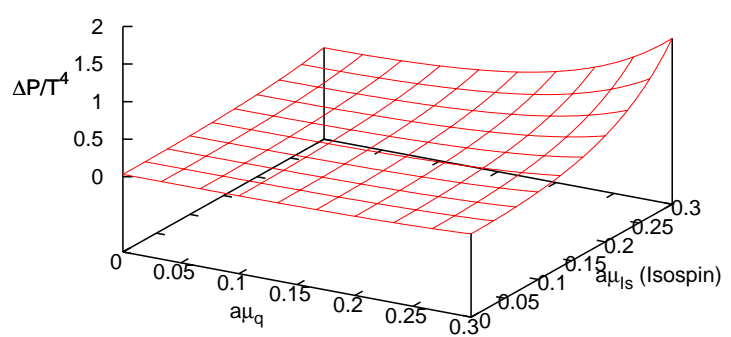

(b)

Figure 3: Equation of state (Pressure) as a function of $a \mu_{q}$ and $a \mu_{I s}$ at (a) $\beta=5.53\left(T / T_{c} \sim 1.1\right.$ ) and at (b) $\beta=4.90\left(T / T_{c} \sim 0.83\right)$.

the systematic stepsize errors caused by the R-algorithm, and found no significant difference for this lattice size and quark mass. At each simulation point we have accumulated 12000 to 20000 measurements. The measurements were taken every 5 trajectories to balance the computational effort of the R-algorithm simultion and measurements.

\subsection{Fitting to $\chi_{i j}$}

We determine $f_{n m}$ by fitting all the derivatives simultaneously to the corresponding ansatz of $\chi_{i j}$. We used the polynomial ansatz eq.(2.4) for the data at $T / T_{c} \geq 0.99$, and the HRG ansatz eq.(2.5) at $T / T_{c} \leq 1.0$. Tables 1 and 2 show the $\chi^{2} /$ dof for the polynomial and HRG fits, respectively. The fitting range of $a \mu_{I}$ is $0.0-0.24$, which covers most of the range up to the RobergeWeiss transition at $\mu_{I}=\pi T / 3$.

Fig. 1 compares the $\chi^{2} /$ dof among various polynomial and HRG fits. One can see that the 4 th order polynomial $((n+m) \leq 4$ in the expansion eq.(2.4)) is not good over the whole temperature range, and that the 6th order one becomes poor in the vicinity of $T_{c}$. Similarly, one can also see that the quality of the fit based on the HRG ansatz becomes poor for $T / T_{c} \geq 0.95$. While the failure of the HRG ansatz near $T_{c}$ has been noticed before [14, 15], it is remarkable that we can see clear indications of 6th order, and even 8th order Taylor coefficients with our modest study. The measurement of 8th order Taylor coefficients represents the current state of the art [10].

Fig. 2 shows the Taylor coefficients $f_{11}, f_{20}, f_{22}, f_{40}$ and $f_{31}$ as a function of temperature. Those results are obtained by fitting a 6th order polynomial in a range of $a \mu_{I}=0.0-0.24$. For $T / T_{c} \geq 1.0$, they agree well with those obtained from the direct measurement of derivatives at $\mu=0$, i.e. $\left.\chi_{i j}\right|_{\mu=0}$, but are more accurate. We do not show the 6 th order Taylor coefficients $f_{60}, f_{51}, f_{42}$ and $f_{33}$ : even though their collective effect is statistically significant, they cannot be individually determined with any statistical accuracy. We only observe that $f_{60}$ is dominant at this order.

Similary, the Taylor coefficients obtained from the HRG ansatz for $T / T_{c} \leq 0.95$ also agree well with direct measurements of $\left.\chi_{i j}\right|_{\mu=0}$, with higher accuracy. However, for $T / T_{c}>0.95$ the results 


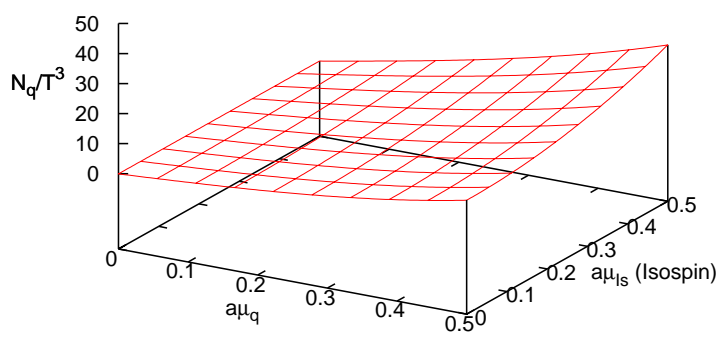

(a)

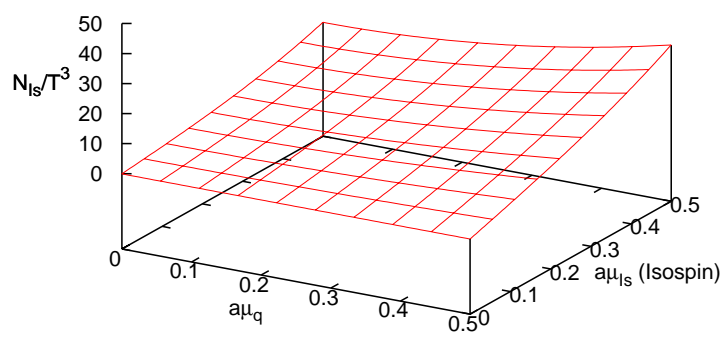

(b)

Figure 4: Number density at $\beta=5.53\left(T / T_{c} \sim 1.1\right)$ as a function of $a \mu_{q}$ and $a \mu_{I s}:$ (a) $N_{q} / T^{3}$ and (b) $N_{I s} / T^{3}$.

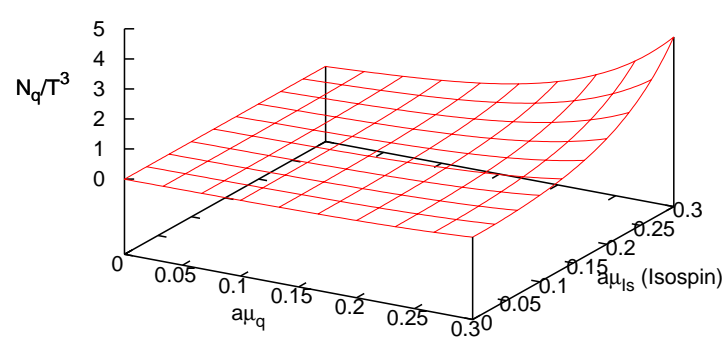

(a)

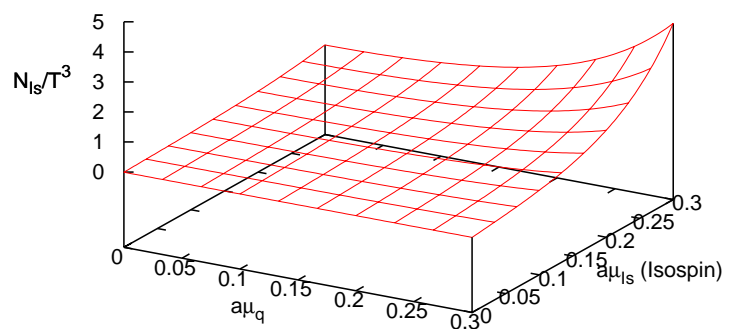

(b)

Figure 5: Number density at $\beta=4.90\left(T / T_{c} \sim 0.83\right)$ as a function of $a \mu_{q}$ and $a \mu_{I s}:$ (a) $N_{q} / T^{3}$ and (b) $N_{I S} / T^{3}$.

from the HRG ansatz fits deviate from $\left.\chi_{i j}\right|_{\mu=0}$. This observation is consistent with the measured $\chi^{2} /$ dof, which increase considerably for $T / T_{c}>0.95$.

\subsection{Equation of State at Finite Densities}

Once we obtain the Taylor coefficients of the pressure or the parameters of the HRG model, we can reconstruct the equation of state. Here, we present two cases at $\beta=5.53\left(T / T_{c} \sim 1.1\right)$ and $\beta=4.90\left(T / T_{c} \sim 0.83\right)$ which are reconstructed with the Taylor series and the HRG ansatz, respectively. Fig. 3(a) shows the equation of state at $\beta=5.53\left(T / T_{c} \sim 1.1\right)$ as a function of $a \mu_{q}$ and $a \mu_{I s}$. Similarly Fig. 3(b) shows the equation of state at $\beta=4.90\left(T / T_{c} \sim 0.83\right)$.

One can also reconstruct other interesting quantities. Fig. 4 shows the quark number density $N_{q}$ and the isospin number density $N_{I s}$ at $\beta=5.53$ as a function of $a \mu_{q}$ and $a \mu_{I s}$. Similarly, Fig. 5 shows $N_{q} / T^{3}$ and $N_{I s} / T^{3}$ at $\beta=4.90$. Here, $N_{q}$ and $N_{I s}$ are defined as $N_{q}=\frac{\partial p}{\partial \mu_{q}}$ and $N_{I s}=\frac{\partial p}{\partial \mu_{I s}}$, respectively. 


\section{Conclusions}

We have performed simulations at imaginary chemical potentials and measured the derivatives of the pressure with respect to $\mu$, at zero and non-zero imaginary $\mu$. By fitting all the derivatives to a polynomial ansatz or an HRG ansatz, we obtained the Taylor coefficients of the $\mu / T$ expansion of the pressure about $\mu=0$. The Taylor coefficients obtained by a polynomial fit for $T / T_{c} \geq 1.0$ agree well with the direct measurement of derivatives at $\mu=0,\left.\chi_{i j}\right|_{\mu=0}$, but are more accurate. Remarkably, we find it impossible to obtain a good fit, at any temperature, without including 6th order derivatives. For $T_{c} \leq T \leq 1.04 T_{c}$, 8th order derivatives are necessary. Thus, our approach may provide a cheaper alternative to the direct measurement of high-order derivatives at $\mu=0$.

Similarly, below $T_{c}$ we observed that the Taylor coefficients obtained by the HRG ansatz deviate from $\left.\chi_{i j}\right|_{\mu=0}$ for $T / T_{c} \geq 0.95$, and the HRG ansatz itself gives a poor description of the imaginary- $\mu$ data. The same observation has been made in [15].

Finally, using the obtained Taylor coefficients we reconstructed the equation of state and the number densities as a function of $\mu_{q}$ and $\mu_{I s}$ up to 4th order.

\section{Acknowledgements}

The numerical calculations were carried out on SX8 at YITP in Kyoto University.

\section{References}

[1] I.M.Barbour et al., Nucl. Phys. Proc. Suppl. 60A (1998) 220.

[2] Z. Fodor and S. D. Katz, Phys. Lett. B 534 (2002) 87 [arXiv:hep-lat/0104001].

[3] S. A. Gottlieb, W. Liu, D. Toussaint, R. L. Renken and R. L. Sugar, Phys. Rev. Lett. 59 (1987) 2247.

[4] S.Choe et al., Nucl. Phys. A698 (2002) 395; Nucl. Phys. (Proc.Suppl.) B106 (2002) 462; Phys. Rev. D65 (2002) 054501.

[5] O. Miyamura, S. Choe, Y. Liu, T. Takaishi and A. Nakamura, Phys. Rev. D 66 (2002) 077502 [arXiv:hep-lat/0204013].

[6] I. Pushkina et al. [QCD-TARO Collaboration], Phys. Lett. B 609 (2005) 265 [arXiv:hep-lat/0410017].

[7] C. R. Allton, S. Ejiri, S. J. Hands, O. Kaczmarek, F. Karsch, E. Laermann and C. Schmidt, Phys. Rev. D 68 (2003) 014507 [arXiv:hep-lat/0305007].

[8] C. R. Allton et al., Phys. Rev. D 71 (2005) 054508 [arXiv:hep-lat/0501030].

[9] R. V. Gavai and S. Gupta, Phys. Rev. D 68 (2003) 034506 [arXiv:hep-lat/0303013].

[10] R. V. Gavai and S. Gupta, Phys. Rev. D 71 (2005) 114014 [arXiv:hep-lat/0412035].

[11] P. de Forcrand and O. Philipsen, Nucl. Phys. B 642 (2002) 290 [arXiv:hep-lat/0205016].

[12] T.Takaishi and Ph. de Forcrand, Europhysics Conference on Computational Physics 2004, Europhysics Conference Abstracts 28D (2004) 240.

[13] M. A. Clark and A. D. Kennedy, Nucl. Phys. Proc. Suppl. 129 (2004) 850 [arXiv:hep-lat/0309084].

[14] M. Cheng et al., Phys. Rev. D 79 (2009) 074505 [arXiv:0811.1006 [hep-lat]].

[15] M. D’Elia and F. Sanfilippo, Phys. Rev. D 80 (2009) 014502 [arXiv:0904.1400 [hep-lat]]. 